\title{
Static WDM Network Planning with TDM Channel Partitioning ${ }^{\star}$
}

\author{
Achille Pattavina $^{1}$, Massimo Tornatore ${ }^{1}$, Alessandro De Fazio ${ }^{2}$, Guido Maier ${ }^{2}$, \\ and Mario Martinelli ${ }^{2}$ \\ 1 Dept. of Electronics and Information, \\ Politecnico di Milano \\ P.za Leonardo da Vinci,32 - 20133 Milan, Italy \\ \{pattavina, tornator\}@elet.polimi.it \\ 2 CoreCom \\ Via Colombo, 81 - 20133 Milan, Italy \\ maier, martinelli@corecom.it
}

\begin{abstract}
In this paper a new solution for the routing, fiber, wavelength and time slot assignment problem (RFWTA) is proposed for a static WDM network. A set of electronic requests characterized by different bit-rates are groomed on lightpaths by a TDM-scheme, designing the logical topology so that the lightpath bandwidth is efficiently exploited. An efficient TDM-based mapping of low granularity electronic connection requests achieves savings in fiber deployment to accommodate a given static traffic. Our analysis comprises two different network layers. First, all the electronic requests are mapped on the logical topology and groomed to obtain the set of lightpath connection requests. Secondly, such requests are routed and fibers and wavelengths are jointly assigned. We adopt the concept of Multihop Lightpath to constrain the number of Time Slot Switching (TSS) operations for each connection request. After introducing our design approach, we discuss the optimization of two case-study networks under various conditions and we analyze the results.
\end{abstract}

\section{Introduction}

Nowadays, the Optical Transport Network (OTN) based on the Wavelength Division Multiplexing (WDM) technology is the well established primary system to carry traffic of the ever-growing Internet and of the other advanced telecommunication applications. An open question is how low-bandwidth digital data flows can efficiently access transmission trunks such as the lightpaths, having very large $(10 \mathrm{Gbps})$ or huge (40 Gbps) capacity. The trivial solution of assigning an entire wavelength-channel capacity to each electronic connection would cause network oversizing with waste of expensive resources.

* This work has been partially supported by the Italian Ministry of Education, University and Research (MIUR) under the FIRB project TANGO. 
Traffic grooming is a new research topic for optical networks, trying to provide methods to design OTNs in which lightpath-bandwidth fractioning is performed in a cost-effective way. The work we are presenting in this paper deals jointly with grooming and physical resource optimization in OTNs.

The paper is organized as follows. In section 2 basic concepts concerning OTN and grooming are reported. Section 3 introduces and explains our heuristic optimization approach to solve the problem. Finally, section 4 is dedicated to the analysis of the results obtained by applying this approach to some case-study networks.

\section{WDM-Network Model Definition}

Let us describe the network environment we are referring to.

At the physical layer, an OTN is composed by WDM transmission links and Optical Cross Connect (OXC) switching nodes arranged according to the physical topology. An OTN offers an optical-circuit connectivity service by setting up lightpaths. Each lightpath is a point-to-point connection between a source and a destination OXC: it can cross other transit OXCs and it requires a wavelength channel per crossed WDM link. The set of all the lightpaths is called logical topology, and it can be represented by a graph having the termination OXCs as nodes and the lightpaths as edges. Resource allocation (WDM channels) to lightpaths is performed by mapping the logical over the physical topology. Since we are considering multifiber OTNs, this operation comprises Routing and Fiber and Wavelength Assignment (RFWA) for all the lightpaths.

The client layer of the OTN is an electronic circuit-switched network whose digital connections have to be hosted by the lightpaths.

The issue of access-to-bandwidth and channel-granularity adaptation between the electronic and optical flows has been taken into account by the International Communication Union (ITU) defining the recent OTN standard [1]. A three-level hierarchy has been introduced to classify the capacity of both lightpaths and client-layer electronic connections. The levels, denoted by $j$, correspond to bit-rates around $2.5(j=1), 10(j=2)$ and $40 \mathrm{Gbps}(j=3)$. A lightpath of a given level can accommodate digital tributaries of equal or lower level via Time Division Multiplexing (TDM). The basic client-bandwidth quantum $(\approx 2.5 \mathrm{Gbps})$ corresponds to a time-slot in a lightpath payload. Thus, e.g. a $10 \mathrm{Gbps}$ digital connection can be carried by a lightpath with $j \geq 2$ by allocating 4 time-slots. The problem of mapping the set of digital connections on the top of the logical topology thus involves time-slot assignment.

Let us now define the problem we intend to solve in this work. The physical topology and the physical link lengths are known: each WDM link is a multifiber cable and each fiber carries a given number of WDM channels. The fiber number on each link is a variable of the problem and has to be dimensioned in order to support the given set of static digital connections characterized by their bit-rate.

The first step to solve it consists in mapping the connections on the logical topology: we suppose in the present work that the logical topology is a full-mesh, 
i.e. a lightpath could be established between any couple of nodes; anyway this approximation does not affect the genarlity of our algorithm. Two grooming strategies have been jointly exploited to obtain an efficient mapping: TDM (following the previously described scheme) and the Time Slot Switching. Each node is equipped to support TSS. TDM technology is used to make a better use of free time slots within wavelengths. Some wavelengths could be already exploited by other connection requests, but not totally filled, so that residual time slots can be exploited by other requests. TSS technology allow the digital request to exploit multihop lightpaths, i.e. to reach their destination by a sequence of edges belonging to the logical topology (let us observe that between two lightpaths composing the multihop path of a connection an opto electronic conversion has to be performed to process different electronic tributaries). We call this first step step TS-mapping. In other words, we can say that TS-mapping is an aspect of the more general problem called traffic grooming $[2,3]$ and comprises the necessity of solving the Logical Topology Design (LTD) problem [4].

The second part of the problem consists in mapping the logical connection requests on a physical topology to minimize the total number of fibers used to transport the requested traffic volume. This is the RFWA (Routing, Fiber, Wavelength Assignment) part of the problem that concerns the physical network planning [5]. In the present work we will consider Virtual Wavelength Path (VWP) networks, in which every node is fully equipped with converters (opaque OXC) so that any incoming optical signal can always be converted on any idle output wavelength [6]. A solution of the planning problem is found only when resources are chosen and allocated for all the optical connections derived by TS-mapping step (rejecting or blocking a connection request is not considered).

Some proposals in literature face the problem from a point of view slightly similar to our approach: in [7] the concept of Super-Lightpath is introduced to reach more than one destination node exploiting the TDM bandwidth of a single lightpath; in [8] the complete problem is efficiently solved in a dynamic scenario, and the authors referred to it as Routing, Fiber, Wavelength Assignment RWTA problem; in [9] and in [10] the problem is solved by a single step approach, anyway no remarks on physical resources utilization are taken into account.

\section{Heuristic Optimization Method for RWTA Problem}

We solve the RWTA problem using two different planning approaches: a novel two-step algorithm, that represents the proposal of this work, and a simple and intuitive algorithm, that will be useful as a term of comparison for our approach.

Our algorithm is divided in two steps: the former routes electronic connection requests on a logical topology (assumed to be full-mesh) providing an efficient mapping of time slots into lightpath bandwidth; the second solves the RFWA part of the problem. Each edge of logical topology corresponds to a lightpath. From now on we will refer to these approach as TOOL B and its distinct steps will be called respectively TS mapping phase and RFWA phase. A second approach has been implemented just to provide a comparison term: this approach does 
not distinguish two different operations, but the algorithm simply routes an optical connection for each electronic request; then it packs the requested timeslot into channel bandwidth using a first fit strategy, finally it eliminates the empty lightpaths and optimizes the fiber number. We will refer to this second approach as TOOL A in the results section.

Electronic connections can be mapped on the logical topology following two paradigm: single hop or multihop From now on, the term lightpath will be used to indicate a single edge in the logical topology, while the term multihop lightpath will refer to a sequence of edges in the logical topology.

\subsection{TOOL B: TS-Mapping Phase}

Let us consider a simple four-node ring to show TS-mapping phase of TOOL B. First of all, logical topology (in this example the associated full-mesh fournode network) is replicated to form a layered graph (we set a high number of replicated layer, compatible with the amount of offered traffic, so that no request will be refused). Obviously, path conversion between different layer is allowed thanks to vertical edges linking the same nodes of the different planes.

Once layered graph has been built, electronic connection requests have to be routed. The first operation is the analysis of connection bit-rate: the different bit-rate have to be converted in term of basilar time-slot granularity. As previously seen, $2.5 \mathrm{Gbps}$ is the basilar unit, then, as far as connection requiring more than one time-slot unit are concerned, we impose all the 2.5 Gbps units associated to the same electronic request to follow the same route. At the end of the process we could choose the fittest bit-rate $(2.5,10$ or 40$)$ on that lightpath. This limitation on the set of admissible transmission bit-rates reflects the actual situation of network operators, that presumably have deployed a set of transmission equipment with fixed bit-rates. So the algorithm have to route electronic connection characterized by 3 distinct admissible granularity in term of time slots: 1,4 and 16 . Now the algorithm has to route this time slot connections

TS mapping metric. The routing on the layered logical topology is carried on using two well-known strategies: the Shortest Path Routing (SPR) and the Least Loaded Routing (LLR)[5].

SPR: Shortest Path Routing routes a connection on the path having the minimum number of hops (so trying to avoid excessively long multihop path). Applying the shortest path algorithm each connection request is routed on the replicated logical topology; if we hypothesize the case of full-mesh logical topology, this step simply assigns the direct link between each couple of nodes to the connection requested by the node couple and corresponds to a ceiling operation on the number of direct lightpaths needed to support direct traffic.

LLR: Least Loaded Routing strategy routes a connections choosing, among the different alternatives, the path having as most congested link the link with minimal load. This approach allows us to distribute the traffic among the network with the aim of avoiding excessively congested links. 
We will show in the result section the performance of this optimization phase using respectively SPR and LLR metric. Let us observe that when two or more paths are characterized by the same weight with respect to the chosen metric, the algorithm routes the requested connection on the path minimizing another metric, the (SPR-L) metric, built with the aim to discourage utilization of lightpaths that would probably use more fiber mileage in the dimensioning step.

It's worth noting that in case of a link of logical topology with residual capacity, the next connection to be routed on that link can: 1) find a sufficient amount of available capacity (in terms of time slots), so exploiting the residual capacity of a pre-existing lightpath, 2) need the allocation of a new lightpath to support its bit-rate.

Minimizing the optical requests. After pre-optimization phase, the algorithm applies an optimization cycle whose aim is to minimize the number of used lightpaths (that is to say the number of links in the logical topology). The sequence of instructions in the optimization phase is the following:

1. Set the integer variable $\mathcal{L}$ to 1

2. Identify among the allocated lightpaths (logical topology links), those having $\mathcal{L}$ spare time slot

3. For each of the previous lightpath:

- temporary delete the previous lightpaths

- try to re-route all the connections owing to the deleted lightpath on the residual capacity of the remaining lightpaths

- if re-routing succeeds for all connections, then delete definitively the lightpath, otherwise restore it

4. increase $\mathcal{L}=\mathcal{L}+1$

5. if $\mathcal{L}$ is larger than $T-1$ exit, otherwise go to step 2 .

$\mathrm{T}$ is the maximum number of time slot supported by a single lightpath. During the re-routing phase, we have also introduced an input parameter $K$ that sets the maximum number of TSS a connection request can cross. Since a logical link corresponds to a lightpath to be physically routed, it is of great importance to take under control the maximum number of hops. The number of hops a multihop lightpath can cross is constrained by the maximum number of Time Slot Switching (in fact $K=H$-1, i.e. with $\mathrm{N}$ admissible Time slot switching operations, a multihop lightpath composed by at most $N+1$ hops can be built). A single TSS operation requires the demultiplexing of WDM signal in its electronic tributaries, the switching of distinct time slots contained in WDM channel, multiplexing of time slots in a new single WDM channel: it is a complex and expensive operation not to be abused.

The cost reduction introduced by this optimization phase thanks to TDM bandwidth utilization can be easily observed in the example shown in Fig 1, where, rerouting the connection $b$ along the residual capacity of lightpaths preallocated to support connections $a$ and $c$, we are able to route two electronic connections exploiting two (and not three) lightpaths in the physical network. 

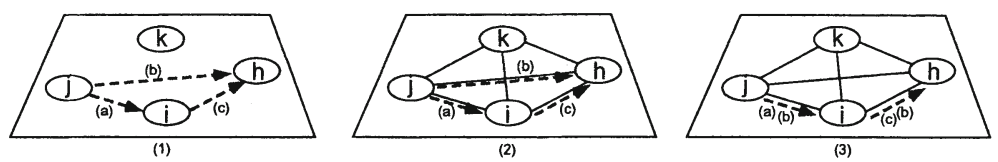

Fig. 1. Re-routing of an electronic tributary (time slots)

Let us assume that up to 16 time slots (according to G.709, 2.5 Gbps $\times 16=$ $40 \mathrm{Gbps}$ ) can be multiplexed on each lightpath. The offered electronic traffic is that shown in Fig. 1(1):

- tributary (a): 1 connection request from node $j$ to node $i$ at 2.5 Gbps

- tributary (b): 1 connection request from node $j$ to node $h$ at 10 Gbps

- tributary (c): 1 connection request from node $i$ to node $h$ at 2.5 Gbps

If we refer to SPR metric, during the first phase of TS-mapping algorithm, connection request are routed on the shortest path, which corresponds in this logical full-mesh topology to the direct link between source and destination node Fig.1(2). So this phase does not involve any TSS operation. Then optimization re-routes electronic flow mapped on $j-h$ lightpath (Fig.1(2)) along the hops $j-i$ and $i-h$ so giving origin to a multihop lightpath as shown in Fig. 1(3). This process allows us to remove a lightpath from optical traffic load, only at cost of the introduction of a TSS operation in node $i$.

Optimization runs are carried on varying the constraint on the number of admissible TSS $(K)$. The increase of admissible cascaded TSS is expected to improve the grooming capability of the algorithm, so influencing the global resource utilization. As a matter of fact, larger values of $K$ provide a wider spectrum of lightpath re-routing alternatives. On the other hand an enlarged set of possibilities is associated to an increase of the network cost, because additional TSS operation costs have to be taken into account.

\subsection{TOOL B: RFWA Phase}

After the optimization phase, the logical topology design is given and provides an optical offered traffic matrix as an input to solve the physical topology design (RFWA). In this matrix the bit-rates are not specified anymore: every lightpath assigned in the logical topology has to be mapped as an optical connection onto physical topology. The algorithm to solve RFWA problem is the same presented in [5], that has proven to provide good performance. It is worth noting that multihop lightpaths will be interpreted as a series of distinct single hop lightpath. For example in Fig. 1(3) the logical topology provides such a optical traffic load:

- 1 connection request from node $j$ to node $i$

- 1 connection request from node $i$ to node $h$

Concluding, the aim of this two-step study (TS mapping associated and followed by RFWA) consists in analyzing the effect of TDM channels partitioning in the mapping of electronic tributaries onto WDM channel, and secondarily in evaluating on its effect on the physical resource utilization. 


\section{Results}

In this section we present and discuss the results we obtained by performing dimensioning experiments on two case-study networks, the EON and the NSFNET. The National Science Foundation Network has 14 nodes and 22 links; the offered traffic matrix ( 360 connection requests distributed on 108 node couples) is taken from [11]. The EON (European Optical Network) is constituted by 19 nodes and 39 links. The offered traffic matrix (1380 connection requests distributed on 342 source-destination node couples) is taken from [12].

Actually, in order to run our experiments, we need an electronic traffic matrix, while in literature we have found traffic matrix listing lightpath requests. So we have adopted the following conversion: each optical connection request is translated in a $10 \mathrm{Gbps}$ unit of bandwidth. This conversion allows us to obtain the value of total bandwidth request per each node-couple requiring connectivity, then we have subdivided randomly the total request between connections characterized by 2.5 and/or 10 Gbps bit-rates. Each fiber is equipped with 16 WDM channels and there are no constraints on the number of fibers a link can support (coherently with our dimensioning approach).

\subsection{ILP Comparison}

First of all, we report the comparison between the performance obtained solving TS-mapping problem by an ILP formulation of the problem and by our algorithm presented in section 3 . We have carried on our experiments on a very simple network case to allow the computationally intensive ILP approach to achieve optimal results in reasonable computational time (seconds). We solve the problem of mapping a dozen of electronic requests on a six-node network: without TSS operation $(K=0)$ the two strategies return the same number of lightpaths needed to support the electronic requests (12); allowing $K=1,2,4$ TSS operations the difference between the number of lightpaths is just one (respectively 9 vs 8 , 8 vs 7,7 vs 6 ). Heuristic results are strictly near to the optimal ones and follow the same trend.

\subsection{SPR vs. LLR in TS-Mapping}

The objective of the TS-mapping in this work consist in minimizing the number of lightpaths necessary to support electronic traffic. We have exploited two distinct approaches to route TS on logical topology before the optimization by means our algorithm. Figures 2(a) and (b) allow us to appreciate the clear performance distance between the two metrics: SPR outperforms LLR for any values of $\mathrm{K}$ parameter and in both networks. We have reported results obtained with $T=16$, anyway a variation on the number of available time slot per frame does not affect this conclusions. It is also possible to notice the positive effect due to introduction of TSS capability on the number of lightpaths needed to support electronic traffic. Let us point out the decrease in the number of connection due to TDM utilization compared with the 360 (1380) electronic requests on NSFNET (EON) case. 


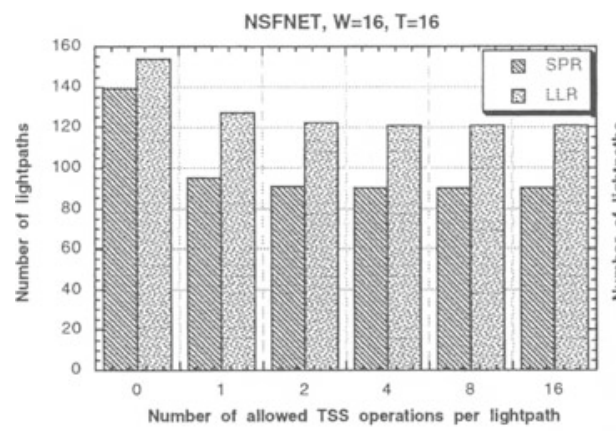

(a)

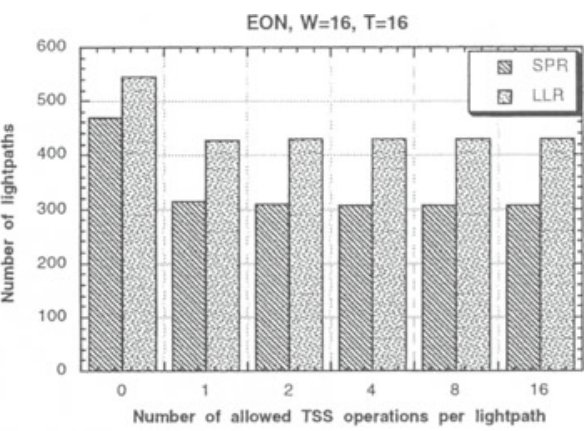

(b)

Fig. 2. Number of lightpaths returned by TS-mapping algorithm varying the number of allowed cascaded TSS on NSFNET (a) and EON (b) topology.

\subsection{T and K: Two Key Parameters}

As previously said in section 3 , the $K$ parameter sets an upper bound on the number of admissible TSS along a multihop lightpath. In our experiments this parameter assumes the values $0,1,2,4,8,16$, where this last value has been set high enough to consider the TS mapping problem solved without any constraint on the number of allowed TSS. Varying this parameter we can investigate the effective trade-off between the additional network equipment complexity and a better resource utilization achievable while allowing an increasing number of admissible cascaded TTS operations.

In Fig. 3 we have plotted the average number of allocated time slots on a single lightpath, varying the number of available time slots on a single channel. Clearly the maximal bandwidth utilization could be represented by a line with unitary slope. We keep in consideration also the RWTA approach referred as TOOL A (see section 3), i.e. the absence of a preliminary and separated TSmapping phase. The first consideration that rises observing this graphic is the relevant advantage in term of network resource usage deriving from the adoption of multihop lightpaths. For increasing values of allowed TSS operation (strictly related to the number of hops of multihop lightpaths), the curves come closer to the ideal one. Then, also this graphic confirm that the practical bound on the number of cascaded TSS to obtain an efficient utilization of channel bandwidth is $K=4$, although even $K=2$ provides quite good performances. Besides, Fig. 3 allows us to compare the TOOL A approach with the TOOL B experiments in case of $K=0$. Both these strategies do not support multihop lightpaths, because TSS operations are not taken in considerations. Nevertheless, TOOL B achieves a better utilization degree than TOOL A. Therefore, we can conclude that the largest gain in term of efficient channel occupation is due to TSS capability, but we have not to neglect that a small contribute is associated to the utilization of a separated TS-mapping phase. This phase provides a preliminary packing of time-slots in wavelength bandwidth that outperforms the on-line packing of 
time slots provided by TOOL A approach, even in the case of absence of TSS capability.

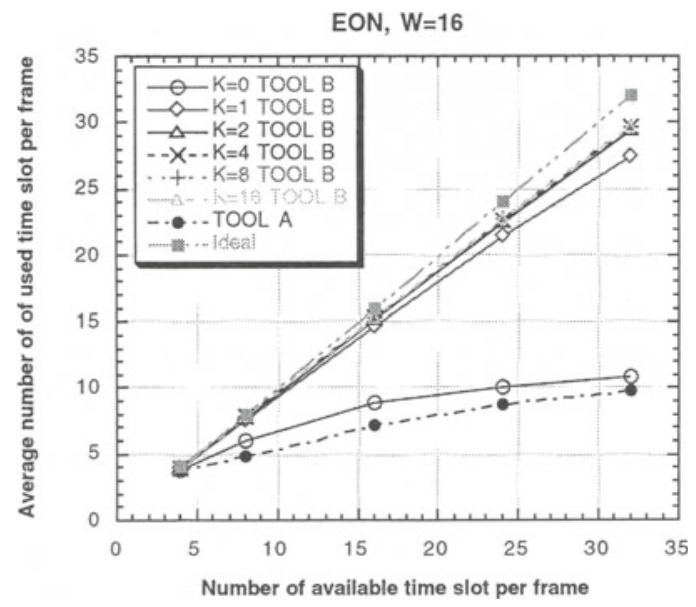

Fig. 3. Average number of allocated time slots per frame varying the number of available time slots, on EON topology.

\subsection{TDM Effects on Fiber Dimensioning}

In this paragraph we show the results obtained thanks to the RFWA step of our analysis. We refer to the number of fibers employed to satisfy all the connection requests in order to provide an estimation of the effect of the introduction of TDM technique on the physical network cost. In Fig.4(a), for the EON network, we have drawn the variation of the total fiber number after dimensioning, varying the number of available time slot per frame, for different values of parameter $K$. The graphic shows that the case without TSS capabilities is much more expensive in terms of fiber cost than the cases in which TSS operations are allowed. A key improvement can be observed between $K=0$ and $K=1$, while there is a modest gap between $K=1$ and $K=2$ and then a further increase of $K$ does not imply any relevant cost reductions. Finally in Fig. 4(b) we plot the dependence of the total fiber number on the number of allowed TSS operations, keeping constant the number of time slot per frame. From this slightly different point of view, it is possible to appreciate the relevant decrement of total fiber number, when (also a small number as 1 or 2) TSS operations are allowed. In particular this graphic points out that TSS utilization is a key-factor especially when the frame supports an high number of time slots. In fact, with a larger number of timeslots per frame, there is a higher probability that request re-allocation succeeds, being available more residual capacity for the optimization algorithm described in the paragraph 3.1. Analogous results has been obtained in NSFNET case. 


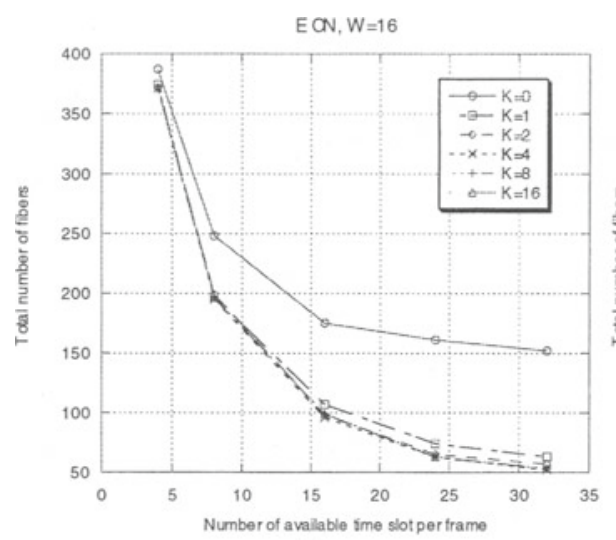

(a)

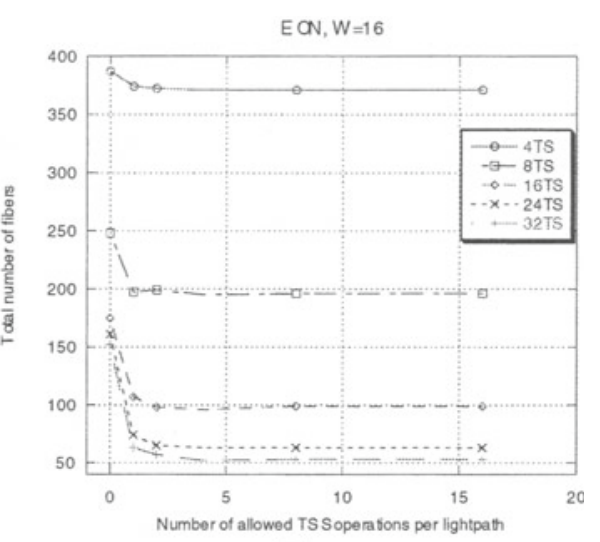

(b)

Fig. 4. (a)Total fiber number varying available time slots per frame, for different values of $K$ and (b)total fiber varying admissible cascaded TSS operation, for different values of $T$, on EON topology.

\subsection{Network Cost Analysis}

In this last paragraph we propose a cost estimation that includes TDM technology equipment in order to provide a cost comparison with network scenario without TDM capabilities (Wavelength Routed, WR).

Let us now introduce a new cost function, alternative to the fiber or channel number: the graphics in Fig. 5(a) and 5(b) have been obtained using the simple cost function in equation (1),

$$
C=F \cdot T
$$

where $F$ is a variable that expresses the number of employed fibers, while $T$ is the number of time slot per frame.

The line WR identifies the cost of dimensioning under wavelength routed hypothesis, i.e. when for each electronic connection request, independently of the bandwidth, a wavelength channel is assigned to the request.

It is not difficult to argue that, when the number of time slot per frame increases (and so the capacity of a single wavelength channel), there is a lower fiber requirement, but at the same time the global network cost is affected by an increment due to the cost of TDM technology and of TSS equipment (i.e. there is an actual cost dependency also by $T$ ). Let us focus our attention on the curve with $K=0$, that shows an increasing trend much steeper than all the other curves, which on the contrary lie in the same order of magnitude. This leads to an important conclusion: TDM technology offers cost performance competitive with traditional WR approach only if TSS capabilities are implemented, providing a technology platform to efficiently exploit multihop lightpaths.

Then, it is worth noting as, in EON case, a network with higher connectivity degree and a larger offered traffic than in NSFNET, the TDM approach results 


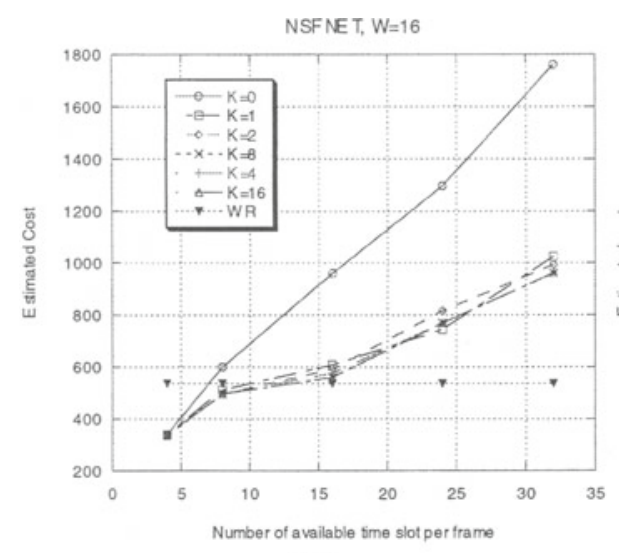

(a)

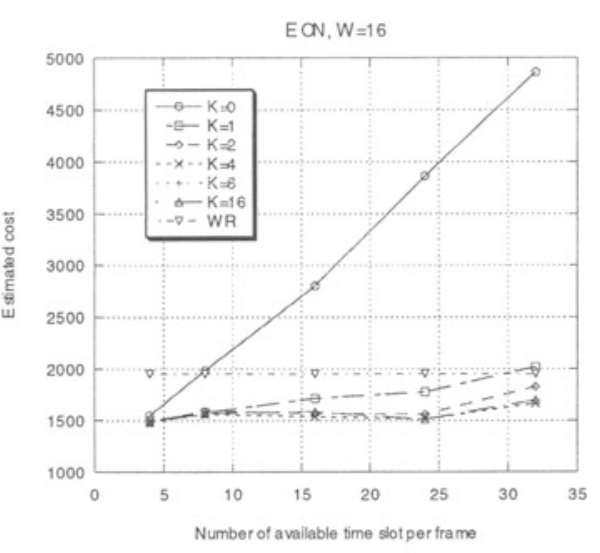

(b)

Fig. 5. Cost function varying available time slots per frame, for different values of $T$, on NSFNET (a) and on EON topology (b).

more cost-efficient thanks to the considerable reduction of fibers, even accepting a unique admissible TSS per connection request. In NSFNET case instead, costs show a steady state till $T=16$ (except the case with $T=0$ whose cost grows rapidly), then they exceed WR threshold reaching higher costs. We can argue that cost convenience in applying TDM channel partitioning strategy to map electronic requests on a WDM networks is in dependence of offered traffic amount (with a higher traffic load, it is possible to save a larger amount of network resource) and connectivity degree.

This work assumes that the number of TSS does not affect the global network cost, so that we observe that cost decreases when the number of admissible TSS increase thanks to an efficient grooming phase. This is a simplifying approximation, but it can be partially acceptable if we consider networks in which every nodes have TSS capabilities already installed, so that additional TSS operation cost is negligible. Besides, this hypothesis has been introduced, because in the grooming phase we impose a constraint on the number of sequential (cascaded) TSS, but we can not decide a priori in which nodes the grooming activity is performed: so all the nodes are assumed to perform TSS. A further development of this work would include an efficient strategy to choose the nodes where TSS capability could be located. Concluding, a cost function, which suitably describes the trade-off between physical resources and TDM processing, is difficult to identify and it follows different criteria according to network features. In this work we have fixed some of these network features, putting in evidence the dependence on network topology (number of nodes, connectivity degree), the volume of offered traffic, time slots per frame and admissible cascaded TSSs. 


\section{Conclusions}

In this work we have developed a new heuristic method aimed at optimizing the number of lightpaths needed to support a given electronic traffic by means of TDM technique. Then we have extended our analysis on the effect of TDM channel partitioning on physical resources. This analysis has been carried out referring to a static traffic matrix and matching the TDM features with the requirements of G.709 ITU-T.

TDM is a key technique to obtain an efficient utilization of wavelength bandwidth. A relevant performance improvement has been achieved exploiting just one or two TSS operations, i.e. allowing the utilization of lightpath multihop to map electronic connections, particularly for high values of time-slots per frame. An approximated cost study, carried out to evaluate the convenience of TDM technique, has highlighted a positive dependence on the traffic load and connectivity degree.

\section{References}

1. Architecture of Optical Tranport Networks (OTN). G.709/Y.1331, Amendment 1. ITU-T International Communication Union (2001)

2. Thiagarajan, S., Somani, A.K.: Traffic grooming for survivable WDM mesh networks. SPIE Optical Network Magazine (2002)

3. Zhu, K., Mukherjee, B.: Traffic grooming in an optical WDM mesh networks. IEEE Journal on Selected Areas in Communications 20 (2002) 122-133

4. Dutta, R., Rouskas, G.N.: A survey of virtual topology design algorithms for wavelength routed optical networks. SPIE Optical Network Magazine 1 (2000)

5. Dacomo, A., Patre, S.D., Maier, G., Pattavina, A., Martinelli, M.: Design of static resilient WDM mesh-networks with multiple heuristic criteria. In: Proceedings, IEEE INFOCOM '02. (2002)

6. S. Chaudhuri, E.B., Ellinas, G.: Addressing transparency in DWDM mesh survivable networks. OFC 20012 (2001) TuO5-1-TuO5-3

7. Mellia, M., Leonardi, E., Feletig, M.: Exploiting OTDM technology in WDM networks. In: IEEE INFOCOM 2002. (2002)

8. B.Wen, Sivalingam, K.: Routing, Wavelength and Time-Slot Assignment in Time Division Multiplexed Wavelength-Routed Optical WDM Networks. In: IEEE INFOCOM 2002. (2002)

9. Zhu, H., Zang, H., K.Zhu, Mukherjee, B.: A Novel Generic Graph Model for Traffic Grooming in Heterogeneous WDM Mesh Networks. IEEE/ACM Transactions on Networking 11 (2003) 1-16

10. Fumagalli, A., Cerutti, I.: Multi-Rate and multi-Hop Hybrid Optical Networks with Arbitrary Topology. In: Proceedings, HPSR '03. (2003)

11. Miyao, Y., Saito, H.: Optimal design and evaluation of survivable WDM transport networks. IEEE Journal on Selected Areas in Communications 16 (1999) 11901198

12. Fumagalli, A., Cerutti, I., Tacca, M.: Survivable Networks Based On Optimal Routing and WDM Self-Healing Rings. In: Proceedings, IEEE INFOCOM '99. (1999) 